\title{
Research Paper \\ Effect of Aerobic Exercise and Kefir Consumption on Anthropometric Indices, Lipid Profile and Blood Pressure in Overweight Non-athletic Women
}

\author{
Azadeh Safari ${ }^{1}\left(\mathbb{1}\right.$, * Mehdi Moradi ${ }^{2}$, Mojtaba Khansooz ${ }^{1}$ (1) \\ 1. Department of Physical Education, Mahallat Branch, Islamic Azad University, Mahallat, Iran \\ 2. Department of Physical Education, Arak Branch, Islamic Azad University, Arak, Iran.
}

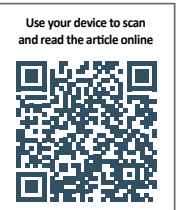

Citation: Safari A, Moradi M, Khansooz M. [Effect of Aerobic Exercise and Kefir Consumption on Anthropometric indices, Lipid Profile and Blood Pressure in Overweight Non-athletic Women (Persian)]. Journal of Arak University of Medical Sciences (JAMS). 2020; 23(2):236-245. https://doi.org/10.32598/JAMS.23.2.4428.3

dol' https://doi.org/10.32598/JAMS.23.2.4428.3

Key words:

Aerobics, Kefir dough, Lipid profile

\section{ABSTRACT}

Article Info:

Received: 17 Sep 2019

Accepted: 01 Feb 2020

Available Online: 01 Jun 2020

Background and Aim The purpose of this study was to investigate the effect of eight weeks of aerobic exercise and the consumption of kefir dough on anthropometric indices, plasma lipid profile and blood pressure in overweight non-athletic women.

Methods \& Materials This quasi-experimental study was performed on 32 non-athletic women with overweight in Arak, Iran (mean age $=33.94 \pm 8.58$ years, mean height $=1.59 \pm 0.05 \mathrm{~cm}$, mean weight $=$ $70.15 \pm 6.88 \mathrm{~kg}$, and body mass index $=27.35 \pm 1.50 \mathrm{~kg} / \mathrm{m} 2$ ) who were voluntarily selected and randomly divided into four groups of 8 (control, exercise, exercise + supplement, and supplement). The control group had a normal diet without exercise; the supplement group received $100 \mathrm{ml}$ kefir dough three meals a day for 8 weeks; the exercise group performed aerobic exercise for 8 weeks, 3 sessions per week, each for 50 minutes. The exercise + supplement group received both aerobic exercise and kefir dough Blood and anthropometric variables were measured at the beginning and at the end of the intervention. Paired t-test was used to examine the within-group variations and one-way ANOVA to examine the between-group variations. All statistical tests were performed at the significant level of $P<0.05$.

Ethical Considerations This study has obtained its ethical approval from the Research Ethics Committee of Qom University (Code: IR.QOM.REC.1398.01).

Results The combination of kefir dough supplementation and aerobic exercise significantly reduced the mean blood pressure $(P=0.003)$ and $B M I(P=0.03)$ but had no significant effect on total cholesterol $(P=0.27)$, triglycerides $(P=0.61)$, high-density lipoprotein $(P=0.16)$, low-density lipoprotein $(P=0.30)$ and waist-to-hip ratio $(P=0.24)$.

Conclusion Overweight women can benefit from the aerobic exercise combined with kefir dough supplementation to reduce their blood pressure and BMI. Further studies are recommended to determine the effect of aerobic exercise and kefir supplementation on serum total cholesterol, triglyceride, low- and high-density lipoproteins, and waist-to-hip ratio.

\section{Extended Abstract}

\section{Introduction}

bese people have harmful fats such as Low-density Lipoprotein (LDL), Total Cholesterol (TC) and Triglyceride (TG) that are above natural. These fats deposit the artery walls and can narrow or block the arteries, resulting in high blood pressure. One of the most effective ways to prevent overweight is to control nutrition (reduce calorie intake) and burn calories by aerobic exercise [4]. The use of herbal supplements and foods in the treatment of diseases and metabolic disorders has become widespread. Kefir is a type

\section{* Corresponding Author:}

Mehdi Moradi, PhD.

Address: Department of Physical Education, Arak Branch, Islamic Azad University, Arak, Iran.

Tel: +98 (918) 8601487

E-mail: mehdy morady59@yahoo.com 
of drinkable yogurt originated in the Caucasus Mountains in the former Soviet Union. This fermented drink is composed of beneficial probiotic bacteria [6]. The aim of this study was to investigate the effect of eight weeks of aerobic exercise and daily consumption of kefir dough on lipid profile, blood pressure and body composition indices in overweight women.

\section{Materials and Methods}

The study population consists of all overweight women living in Arak, Iran with a Body Mass Index (BMI) equal to or greater than $25 \mathrm{~kg} / \mathrm{m}^{2}$, who had no other sports activity except daily physical activity. Subjects were randomly divided into four groups of control, exercise, supplement, and exercise + supplement. The control group had a normal diet without exercise; the supplement group received 100 $\mathrm{ml}$ kefir dough three meals a day for 8 weeks; the exercise group performed aerobic exercise for 8 weeks, 3 sessions per week, each for 50 minutes. Each session consisted of 10 min warm-up and 30 minutes of main exercise. To examine the biochemical variables, blood samples were taken from the subjects 24 hours before the first training session and after the last training session. LDL, High-Density Lipoprotein (HDL) and TG levels were measured using photometric method and enzymatic assay was used for measuring the TC level. The data were analyzed in SPSS software where the Kolmogorov-Smirnov test was used to determine the normality of the data distribution, one-way ANOVA for assessing between-group differences, and paired t-test for evaluating within-group differences.

\section{Results}

The demographic and physiological characteristics of participants are presented in Table 1.

The results showed that HDL concentration increased in all groups, but this increase was not significant in any group. The LDL level decreased significantly in two groups of exercise and exercise + supplement. The ANOVA results showed no significant difference between groups in terms of HDL and LDL levels ( $\mathrm{P} \geq 0.05)$. The TG level in the exercise group increased by $18 \%$, while it was decreased in the exercise + supplement group by $6 \%$. The TC levels increased by about $4.3 \%$ in the exercise + supplement group, but decreased by $4.6 \%$ in the supplement group. These differences in the TC and TG levels were not significant $(\mathrm{P} \geq 0.05)$. According to the one-way ANOVA results, there was no significant difference between groups in terms of TG $(\mathrm{P}=0.55)$ and TC $(\mathrm{P}=0.09)$. The mean blood pressure decreased in most subjects, where the highest reduction was reported in the exercise + supplement group (10\%). This reduction was significant in the exercise + supplement $(\mathrm{P}=0.003)$ and exercise $(\mathrm{P}=0.007)$ groups; however, it was no significant between groups $(\mathrm{P}=0.74)$. The $\mathrm{BMI}$ value was reduced in all groups, but it was significant only in the two groups of exercise $(\mathrm{P}=0.04)$ and exercise + supplement $(\mathrm{P}=0.03)$. Based on the one-way ANOVA results, there was no significant difference between the groups in terms of BMI ( $\mathrm{P}=0.51)$. Regarding the Waist-to-Hip Ratio (WHR), there was no significant difference between the pre- and post-test scores of the groups $(\mathrm{P} \geq 0.05)$, and between study groups $(\mathrm{P}=0.52)$.

\section{Discussion}

The results of the present study show that aerobic exercise alone had significant effects on LDL and blood pressure levels, but it had no significant effect on the HDL,TC and TG levels. The TC and TG levels were reduced after 8 weeks of exercise, but the difference between pre- and post-test scores was not significant. This is consistent with results of Jurima et al [8]. They reported that the TC and TG levels did not change significantly after 24 weeks of exercise compared to baseline values. They suggested that it might because of insufficient exercise intensity. Zolads et al. suggested that, in order to increase HDL level, exer-

Table 1. Mean \pm SD of demographic and physiological characteristics

\begin{tabular}{ccccc}
\hline Characteristics & Control & Supplement & Exercise & Exercise + Supplement \\
\hline Age & $34.50 \pm 9.94$ & $33.12 \pm 6.79$ & $30.12 \pm 4.99$ & $38.00 \pm 12.60$ \\
\hline Height $(\mathrm{m})$ & $1.61 \pm 0.06$ & $1.62 \pm 0.05$ & $1.59 \pm 0.05$ & $1.57 \pm 0.07$ \\
\hline Body fat percentage (\%) & $30.42 \pm 2.81$ & $30.17 \pm 4.27$ & $26.03 \pm 1.52$ & $30.37 \pm 6.71$ \\
\hline BMl (kg/m2) & $26.87 \pm 1.21$ & $28.04 \pm 1.93$ & $26.93 \pm 0.98$ & $27.57 \pm 1.91$ \\
\hline Weight $(\mathrm{kg})$ & $70.25 \pm 7.04$ & $73.5 \pm 4.53$ & $68.50 \pm 7.19$ & $68.37 \pm 8.76$ \\
\hline
\end{tabular}


cise should be done daily and with sufficient intensity and duration [9]. The present study reported that kefir dough alone had a significant effect on the LDL level. Despite the reduction in HDL, TC and blood pressure observed in the exercise + supplement group, the difference between the two groups was no significant. Consumption of kefir along with aerobic exercise caused a significant difference in blood pressure between the pre-test and post-test scores, but between groups, the change was significant changes only in terms of the maximum oxygen consumption and body fat percentage. Consumption of kefir along with aerobic exercise caused significant difference in weight and BMI between pre- and post-test scores, but there were no significant difference between study groups.

\section{Conclusion}

It seems that overweight women can use aerobic exercise combined with kefir dough supplementation to reduce their hypertension and BMI. Further studies are recommended on the effects of aerobic exercise and kefir dough supplementation on WHR, HDL, LDL, TG, and TC.

\section{Ethical Considerations}

\section{Compliance with ethical guidelines}

This study has obtained its ethical approval from the Research Ethics Committee of Qom University (Code: IR.QOM.REC.1398.01)

\section{Funding}

The present paper was extracted from the MSc thesis of the first author (Code: 2002,1404941013), Department of Physical Education, Mahallat Branch, Islamic Azad University.

\section{Authors' contributions}

All authors met the writing standards based on the recommendations of the International Committee of Medical Journal Editors (ICMJE).

\section{Conflicts of interest}

The authors declared no conflict of interest. 


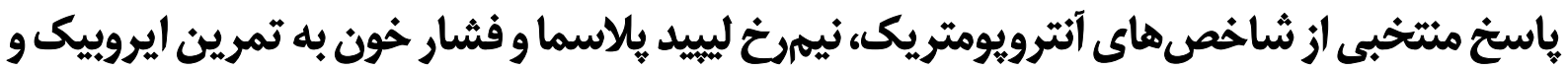

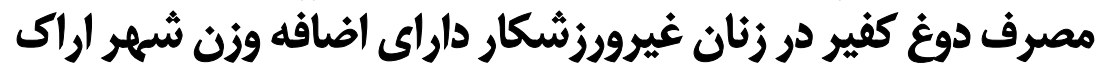

آزاده صفرى' هـ، "مهدى مرادى 'م، مجتبى خانسوز'

I. كروه تربيتبدنى، واحد محلات، دانشكاه آزاد اسلامى، محلات، ايران.

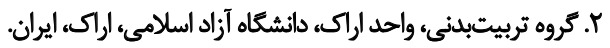

\begin{abstract}
سكيد

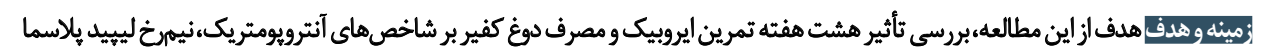

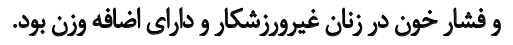

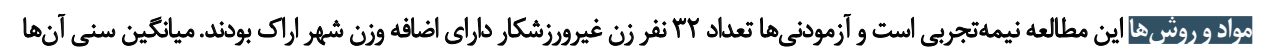
به

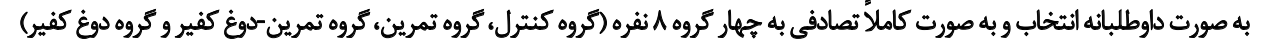

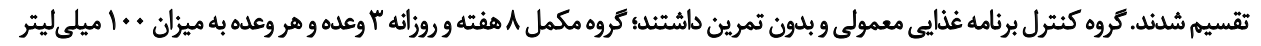

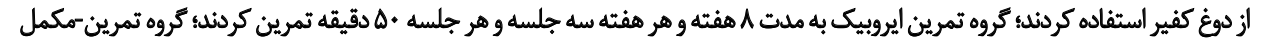

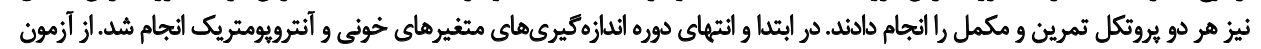

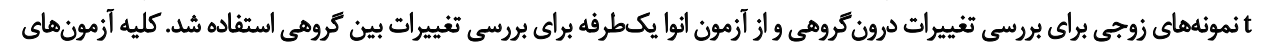

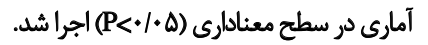

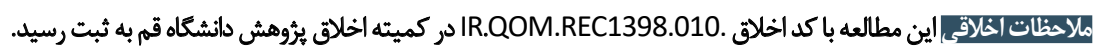

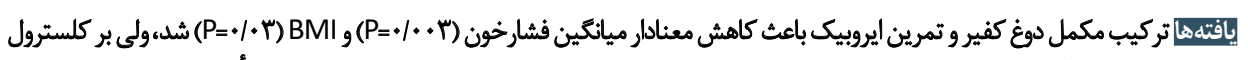

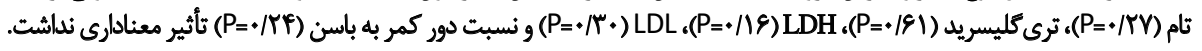

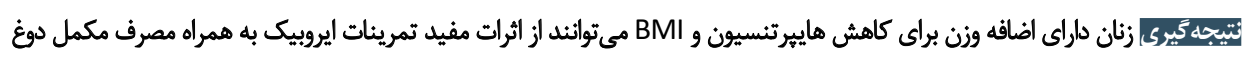

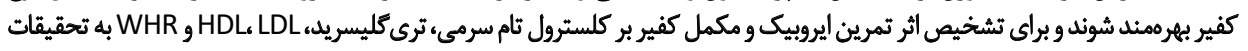
بيشترى نياز است.
\end{abstract}

اطلاعات مقاله:

تاريخ دريافت: و ش شهريور

تاريخ هذيرش: Tا بهمن

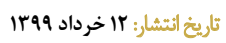

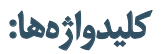

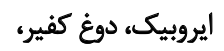
نيهرخ ليبيدى دير كنيرن

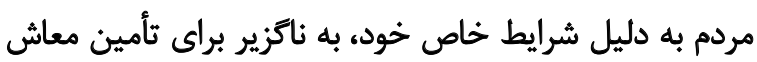

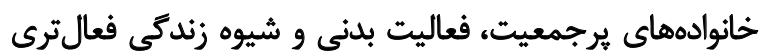

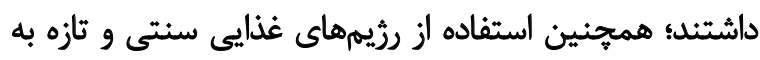
جاي غذاهاى رايج سرخ كرده و آماده كنونى و خواب شائ شبائه بيشتر

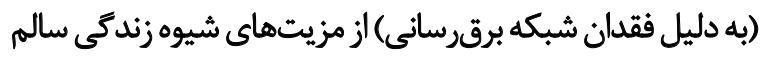

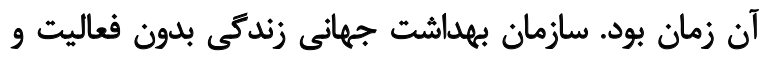

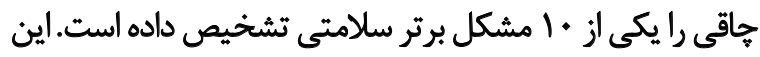

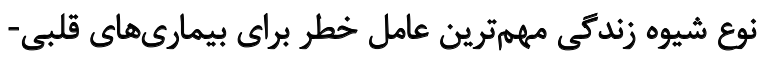

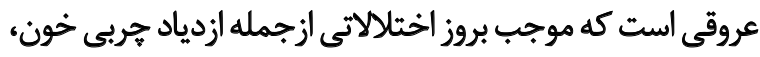

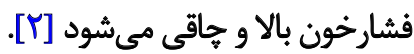

جاقى علت اصلى بسيارى از بيمارىهاى مزمن از قبيل

1.World Health Organization

dateo

اصلى ترين علت جاقى، عدم تعادل بين انرزى دريافتى و انرزى دافى

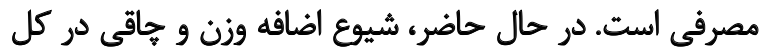

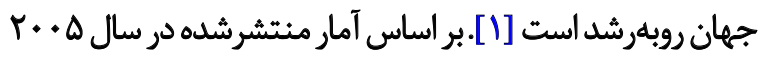

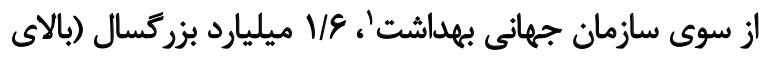

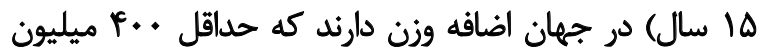

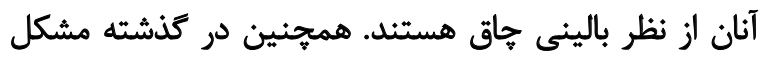

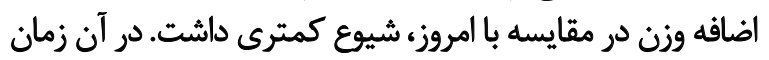

\section{-}

* نويسئده مسئول:

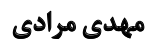
نشائى: اراك، دائشعاه آزاد اسلامى، واحد اراك، كروه تربيتبدينى. تلفن: mehdy_morady59@yahoo.com بـث الكترونيكي: 


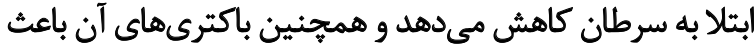

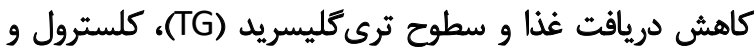

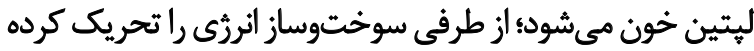

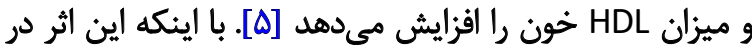

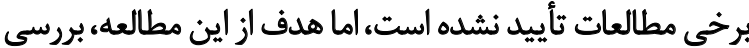

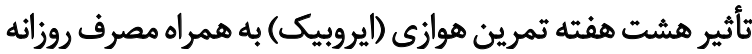

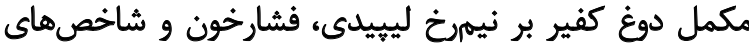
تركيب بدنى در زنان داراى اضافه وزن است.

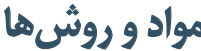

روش كار

مطالعه حاضر در قالب طرح نيمهتجربى است و به صورت

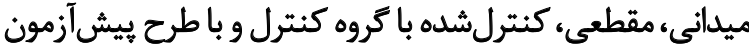

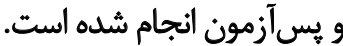

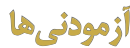

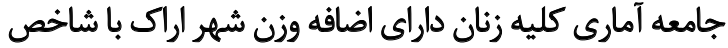

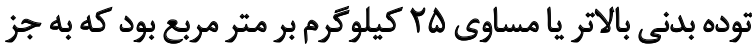

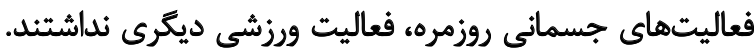

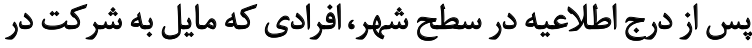

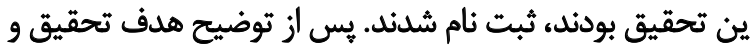
روش كار، با كرفتن

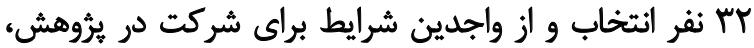

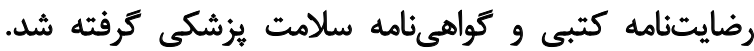

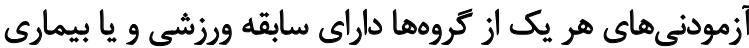

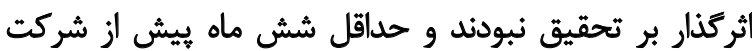

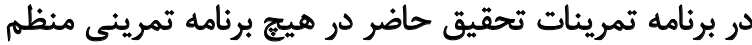

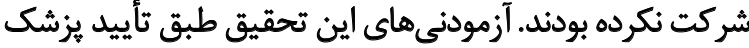
از سلامت جسمانى كامل برخوردار بودند.

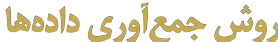

ابتدا هدف، جزئيات و همجنين خطرات احتمالى اجراى

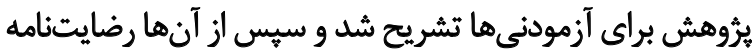

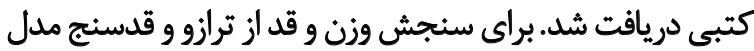

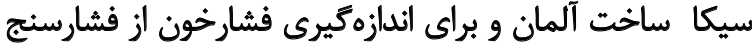

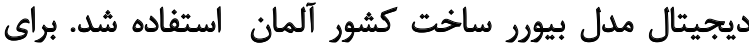

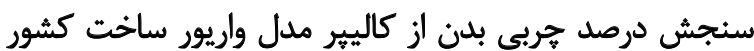

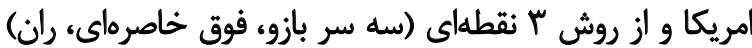

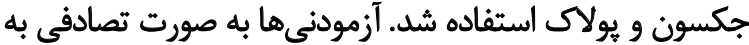

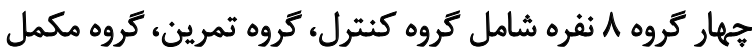

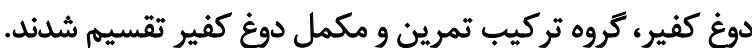

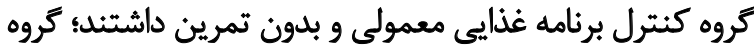

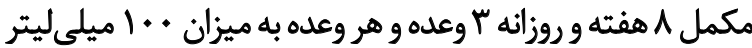

ديابت، فشارخون و بيمارىهاى قلبى است و به دليل بار مالى

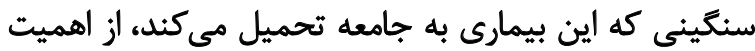

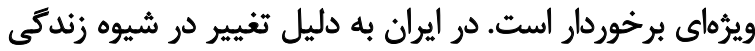

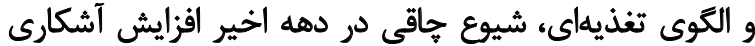

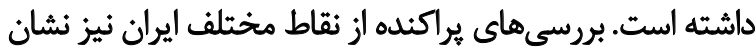

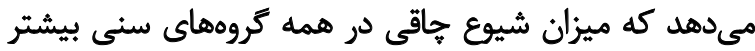

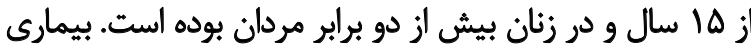

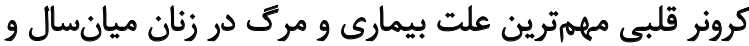

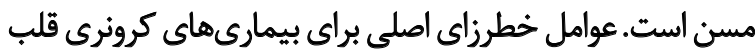

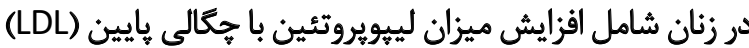

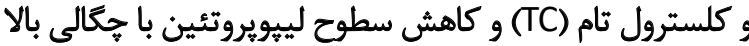

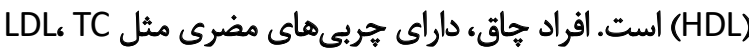

$$
\text { و تريكليسريد (TG) بيش از حد طبيعى هستند. }
$$

اين هربيها در ديواره ركها رسوب كرده و موجب انسداد

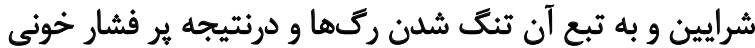

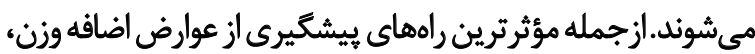

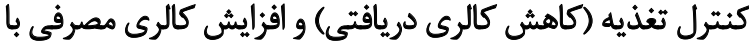

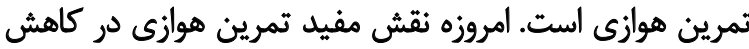

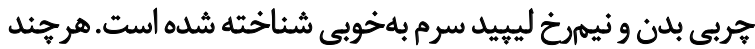

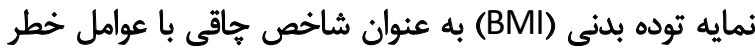

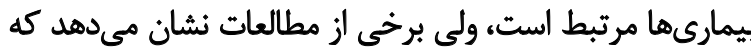

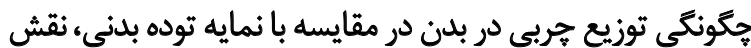

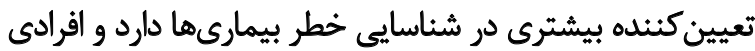

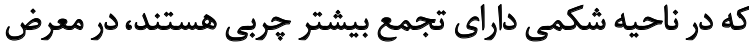

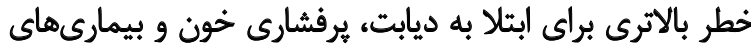

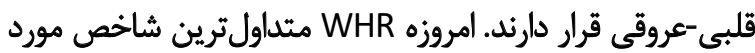

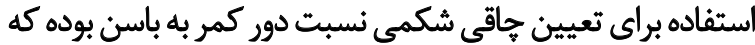

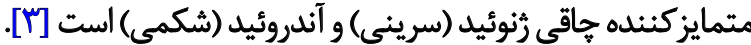

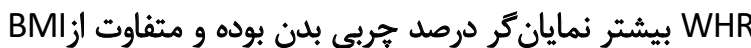

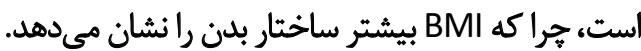

امروزه استفاده از مكملها و غذاهاى كياهى نيز در درمان

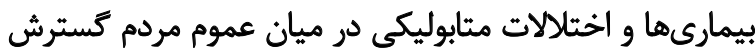

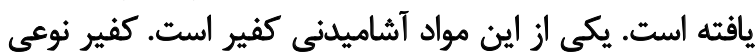

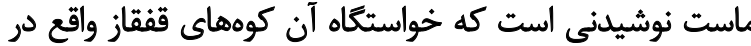

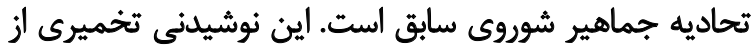

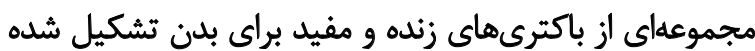

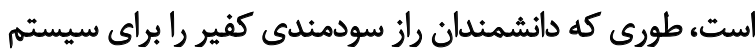

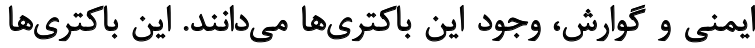

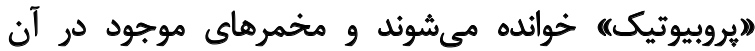

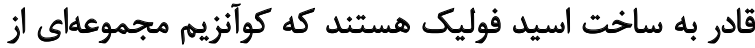

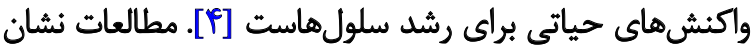

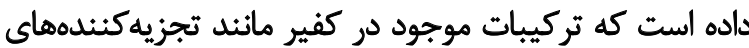

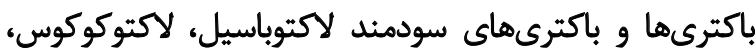
لوكونوستوك، استوباكتر به دليل يروبيوتيك بودن بآينوين اين محصول، لاكتوكي، 


\section{روش آلمارى}

اطلاعات به وسيله نرمافزار SPSS تجزيه و تحليل شد و نتايج

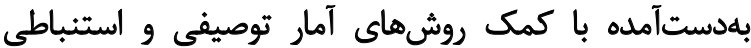

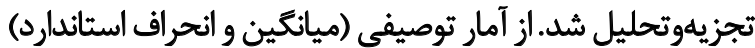

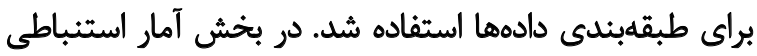

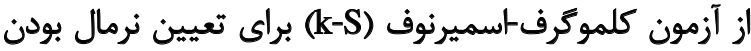

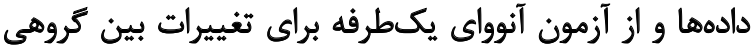

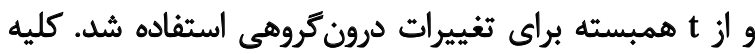

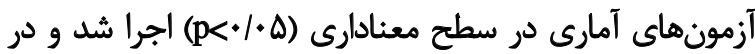
قسمتهاى معنادار از آزمون تعقيبى توكى استفاده شد.

\section{يافتهها}

شاخصهاى دموكرافيك و فيزيولوريكى كروهها در جدول

$$
\text { شماره آ آورده شده است. }
$$

ابثدا مشخص شد كه تفاوت معنادارى در بيش آزمون كروهها

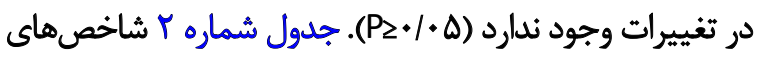

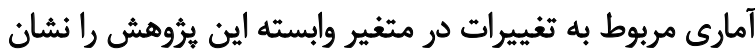

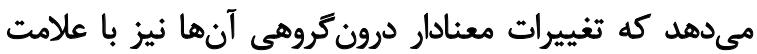

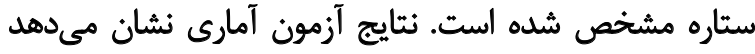

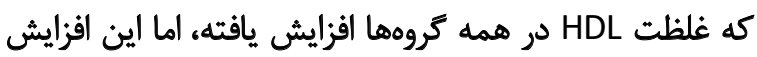

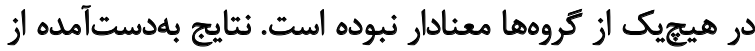

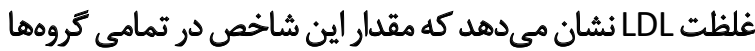

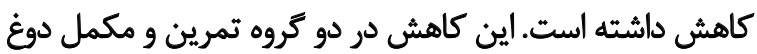

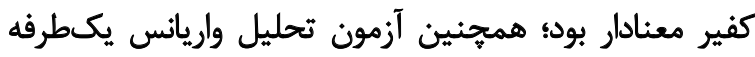

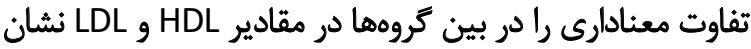

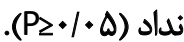

تغييرات TG و TC بلثترتيب در جدول شماره Y بآمده است.

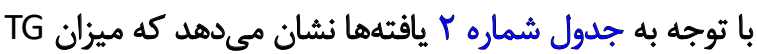

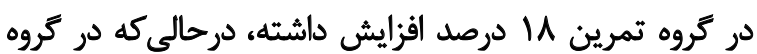

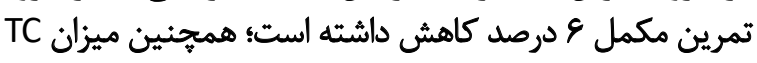

از دوغ كفير استفاده كردند؛ گروه تمرين ايروبيك به مدت م هفته

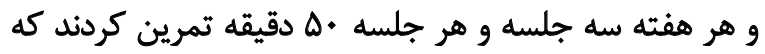

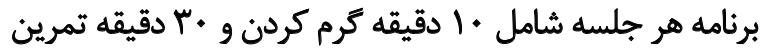

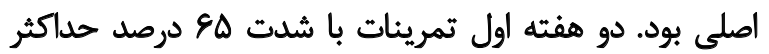

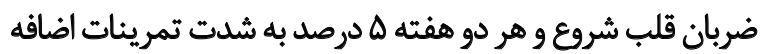

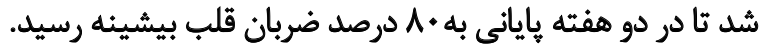

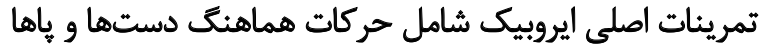

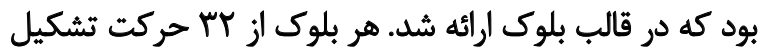

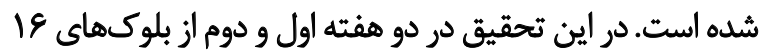

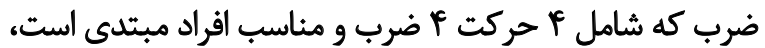

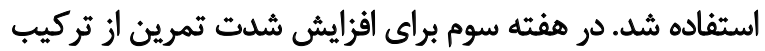

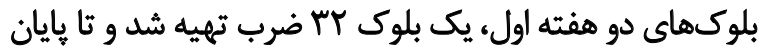

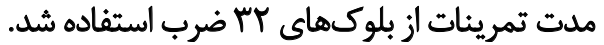

ضربان قلب آزمودنى هاو شدت تمرين بر اساس حداكثر ضربان

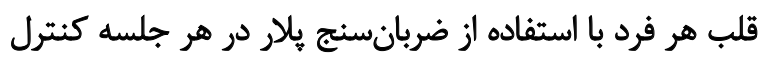

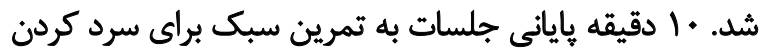

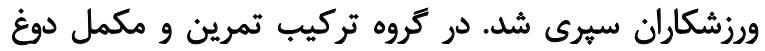

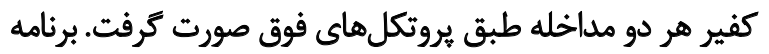

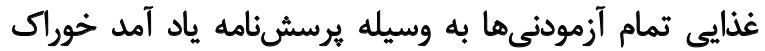

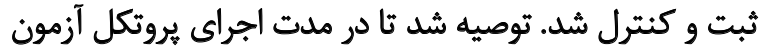

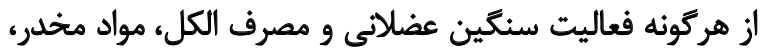

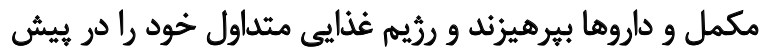

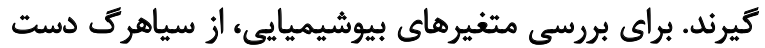

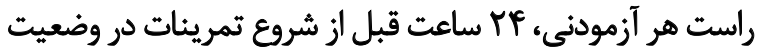

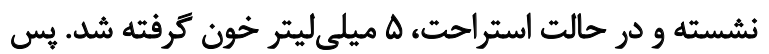

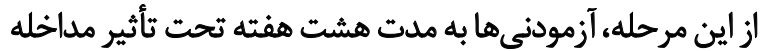

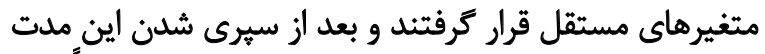

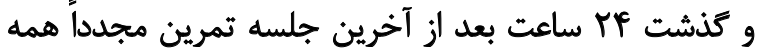

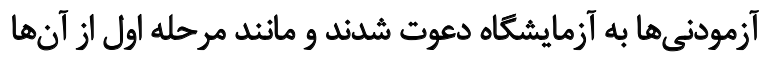

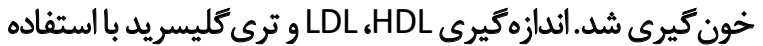

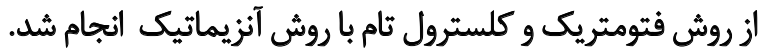

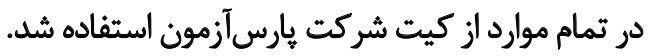

جدول ا. ميانكين و انحراف معيار خصوصيات دموكرافيك و فيزيولوريك سن، قد، درصد جربى بدن، شاخص توده بدنى و وزن

\begin{tabular}{|c|c|c|c|c|}
\hline \multicolumn{4}{|c|}{ ميانكين士انحرافمعيار } & \multirow{2}{*}{ كروههاي مطالعه ـ شاخصهاى مورد مطالعه } \\
\hline تمرين و مكمل & تمرين & مكمل & 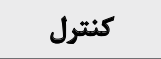 & \\
\hline TNIYE+./F. & $r \cdot / f \pm / r / q q$ & $r / q \pm I T / v a$ & $m e / q \pm \Delta . / 9 \varphi$ & سن (سال) \\
\hline$V / \bullet \pm \Delta V / \bullet V$ & $1 / \cdot \pm \Delta q / \cdot \Delta$ & $1 / \cdot \underline{ \pm} \boldsymbol{e} / \cdot \Delta$ & $1 / . \pm 81 / \cdot 8$ & قد (متر) \\
\hline$r+\mid s \pm r v / M$ & 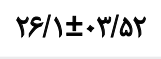 & $r \cdot / \Psi \pm I V / T V$ & $r+/ Y \pm r Y / A I$ & درصد هربيى بدن (درصد) \\
\hline$r V /( \pm \Delta Y / q)$ & $r g / . \pm q r / q$ & $r N \backslash \pm+F / 9 T$ & $r E / I \pm A V / T I$ & شاخص توده بدنى (كيلوكرم بر مترمبع) \\
\hline SNA & $9 N / \pm \Delta \cdot / 19$ & $n / \mu \pm \Delta \cdot / \Delta \mu$ & $V+/ V \pm r \Delta /+r$ & وزن (كيلوكرم) \\
\hline
\end{tabular}


مطالعه مهمترين علل عدم تغيير در شاخصها را ناكافى بودن

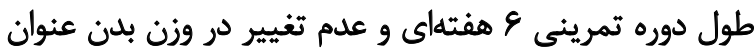

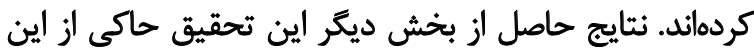

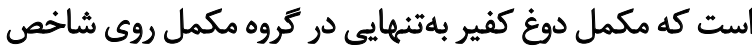

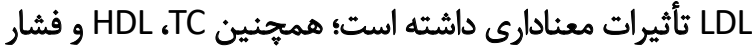

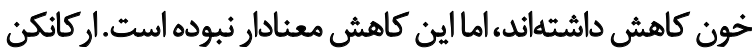

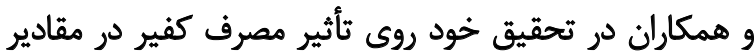

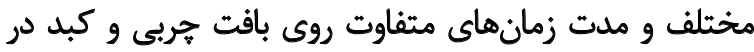

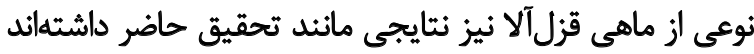

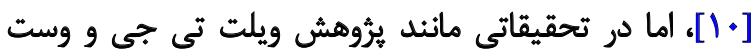

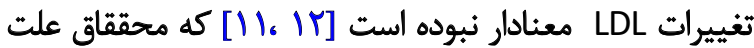

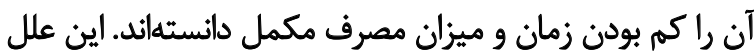

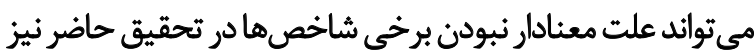

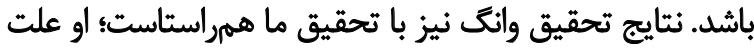

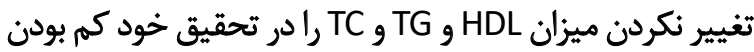

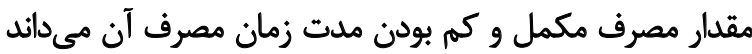

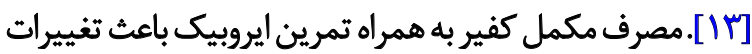

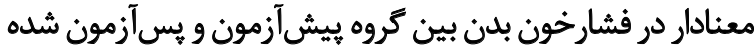

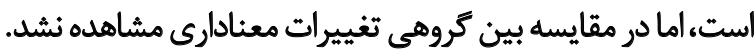

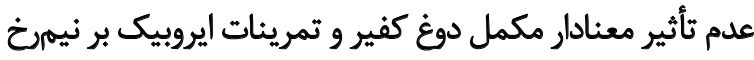

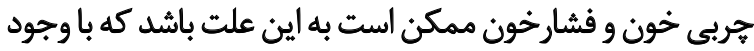

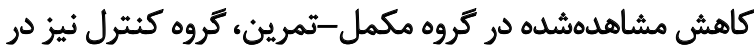

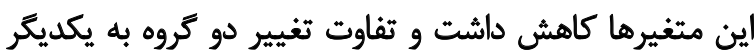
نزديك شد و نتايج معنادارى حاصل نشد.

كلى ويرجينيا در تحقيقى با عنوان لاتأثير مصرف كفير پانس

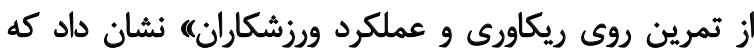

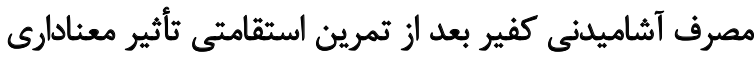

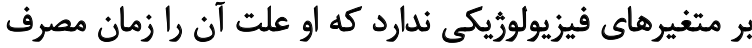

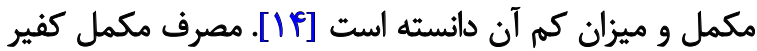

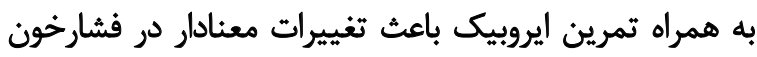

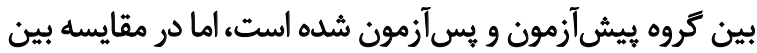

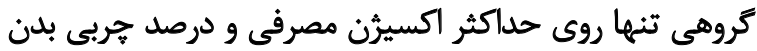

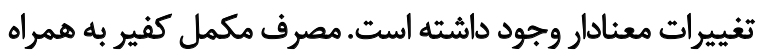

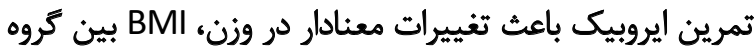

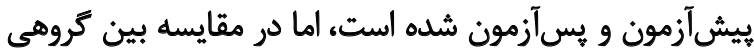

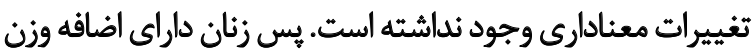

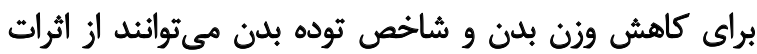

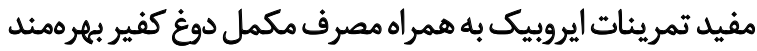

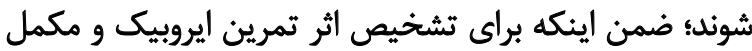
دوغ كفير بر WHR به تحقيقات بيشترى نياز است.

يافتههاى كلثوم بهرامى و همكاران نشان داد كه ثأثير تمرينات

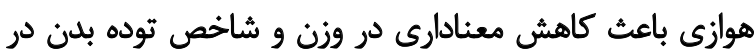

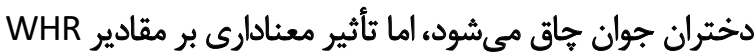

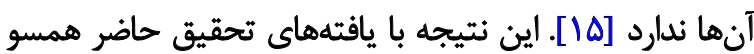

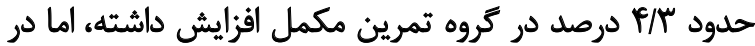

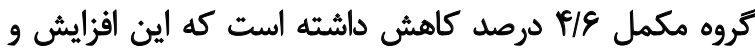

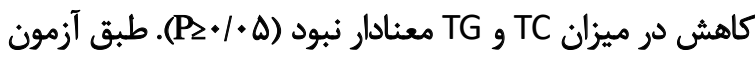
تحليل واريانس يكطرفه هيج تفاوت معنادارى بين كروههاي وجود ندارد. تغييرات فشارخون

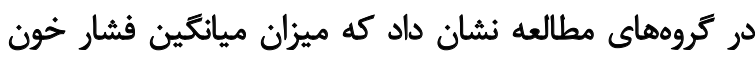

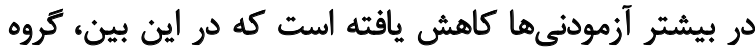

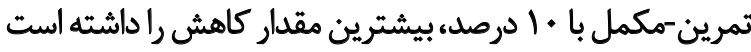

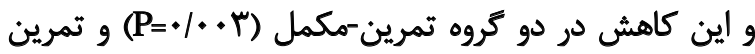

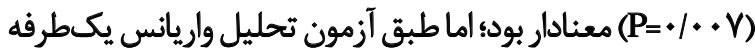

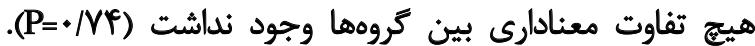

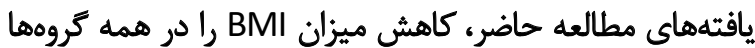

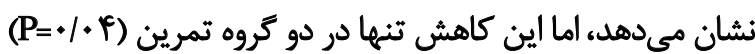

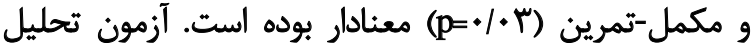

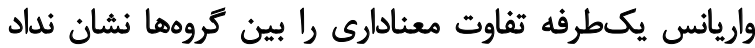

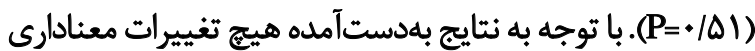

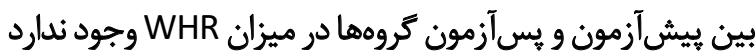

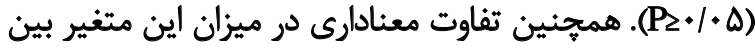
كروها مشاهده نشد (P) (P) (P)

بحث

نتايج ثروهش حاضر نشان مىدهد كه تمرينات ايروبيك

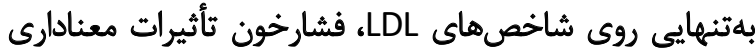

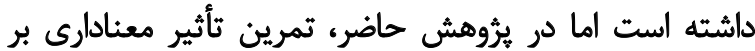

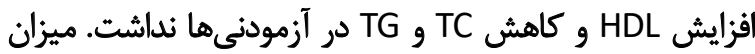

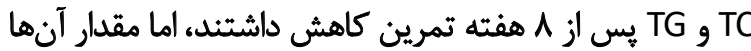

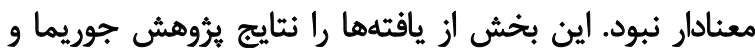

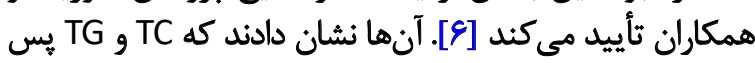

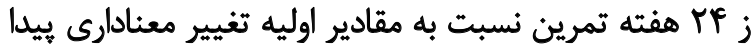

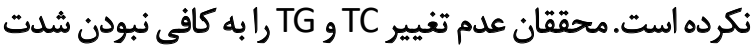

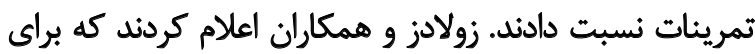

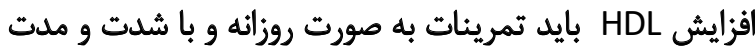

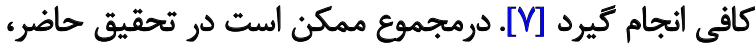

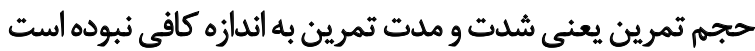

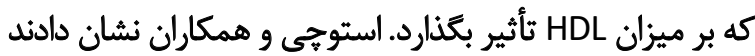

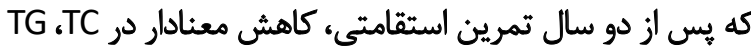

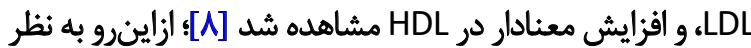

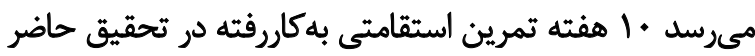

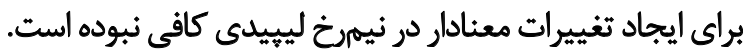
محمدنيا احمدى و همكاران نيز با مطالعه روى موشهاي

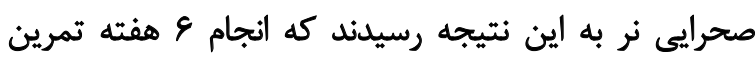

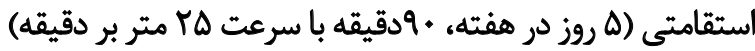

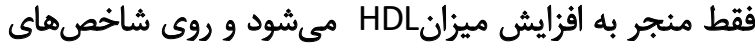

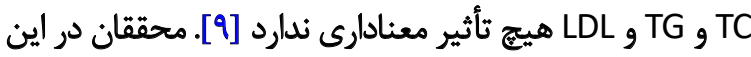


جدول r. ميانكين و انحراف معيار شاخصهاى مورد مطالعه همراه با نتايج آزمون تى و آزمون آنوا

\begin{tabular}{|c|c|c|c|c|c|c|c|}
\hline \multicolumn{2}{|c|}{ آزمون ANOVA } & \multicolumn{2}{|c|}{ 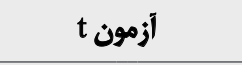 } & \multicolumn{2}{|c|}{ ميانكين 土|نحراف معيار } & \multirow{2}{*}{$\log ^{\prime}$} & \multirow{2}{*}{ شاحصدهاي مطالعه } \\
\hline $\mathbf{P}$ & $F$ & $P$ & $\mathrm{t}$ & يَيسآزمون & يبش آزمون & & \\
\hline \multirow{5}{*}{.100} & \multirow{5}{*}{$r / N$} &.$M 11$ & $-V / A Y$ & $P Q / 1 \cdot \pm A V / T$ & $|p| \cdot . \pm 1 \cdot 189$ & كتثرل & \multirow{5}{*}{ 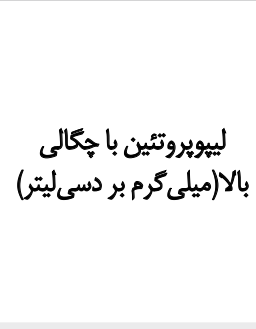 } \\
\hline & &.$/ r$ & $-1 / 1 \% 9$ & $\varphi q / \Delta \pm \Delta \cdot / \pi$ & $r r / N \Delta \pm N R q$ & تمرين & \\
\hline & & $\cdot / r$ & $\cdot / 4 q$ & $\Delta V / 1 \cdot \pm+\cdot / g T^{n}$ & $\Delta V / A V \pm V / q \Psi$ & مكمل & \\
\hline & & .118 & $-V / \Delta r$ & $\Delta Y / A \pm T \Delta / P F$ & $P Q / T V \pm I Y / M T$ & هكمل-تمرين & \\
\hline & & & & $\geq 0$. & & مقدار نرهال & \\
\hline \multirow{5}{*}{.11} & \multirow{5}{*}{$T / M I$} & . IFa & . $M$ & 1. T/MAET/M & $1.8 / 19 \pm 0.181$ & كتترل & \multirow{5}{*}{ لييويروتئين با جكَالى يايين } \\
\hline & & $.11^{*}$ & $m / p e$. & $\Delta F / N \pm \Delta \cdot / M^{e}$ & $9 . / 1 r \pm r / r g$ & تمرين & \\
\hline & & $.1 *$ & rples & จท/. $\pm A \vee / M^{\circ}$ & $1 W / \mathrm{Ir} \pm \mathrm{AV} / \mathrm{F} \Delta$ & مكمل & \\
\hline & & $\cdot / r$ & $\mathrm{M}$ & $9 \% / / F \pm 1 \% / 1$. & WIAETQ/IT & هكمل-تمرين & \\
\hline & & & & <ir. & & مقلار ترمال & \\
\hline \multirow{5}{*}{$\cdot 1 \Delta \Delta$} & \multirow{5}{*}{$+N$} & $\cdot M^{\infty}$ &.$- / m$ & QY/FF $\pm r V / M$ & 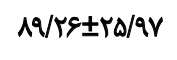 & كتشرل & \multirow{5}{*}{ ترى كليسريد (ميلي كرم بر } \\
\hline & &.$/ M A$ & $-1 / 18$ & Q./ertrv/e & $V \notin / M I \pm \Delta \cdot / r$ & تمرين & \\
\hline & &.$/ \Delta \Delta$ & $-+|8|$ & $\Lambda \& / T \Delta \pm \Delta+/ \Delta q$ & $\lambda r / r+ \pm r V / M$ & 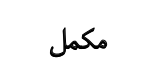 & \\
\hline & & $.18 \mid$ & $\cdot / \Delta r$ & $\Delta F / r+ \pm \cdot \cdot 1 \cdot \Delta$ & $\Lambda 9 / T \Delta \pm+* / \Gamma$ & مكمل-تمرين & \\
\hline & & & & $<r+$. & & مقدار نرمال & \\
\hline \multirow{5}{*}{.1 .9} & \multirow{5}{*}{ r/re } &.$/ M r$ & - - - & $|A| / T P \pm . . / V \Delta$ & $1 E q / r V \pm A V / R r$ & كتترل & \multirow{5}{*}{ كلسترول تام (ميلى كرم بر } \\
\hline & &.$/ r$ & $M$ & $|\Delta F / T Y \pm Y \Delta / T|$ & $1 \Delta V / 19 \pm \Delta \cdot / \Delta P$ & تمرين & \\
\hline & &.$/ 19$ & I/PY & $I V / / \Delta \pm V \Delta / \neg Y$ & WVIIF $\pm I Y / F V$ & مكمل & \\
\hline & & $\cdot / r V$ & $-1 / 11$ & $\mid V \cdot / T \Psi \pm V D / I r$ & $\mid F \Delta / I V \pm I Y / Y Q$ & مكمل-تمرين & \\
\hline & & & & $<r+$. & & مقدار طبيعى & \\
\hline \multirow{4}{*}{$\cdot M^{e}$} & \multirow{4}{*}{$\cdot \mid+i$} & $\cdot \mid M I$ & $1 / \pi \Delta$ & $M V \pm V Y / \cdot \varepsilon$ & $9 Y / / Y \pm$ VNTE & كنترل & \multirow{4}{*}{ فشارخون (ميلمير جيوه) } \\
\hline & & $+1 \cdot+r^{*}$ & $r / N$ & $9 r / \cdot \pm E r / \Delta r$ & WITEFI/M & تمرين & \\
\hline & & $\cdot 1+\Delta$ & $r / \pi r$ & $N / N \pm Q \Delta / 1 E$ & $q \Psi / L \cdot \pm+r / \Delta T$ & مكمل & \\
\hline & & $.1 \cdot+r^{*}$ & $F(\Delta)$ & $9 \cdot 11 \pm F \in M^{F}$ & $1 . . / 1 m \pm m v / u$ & مكمل-تمرين & \\
\hline \multirow{4}{*}{.101} & \multirow{4}{*}{$\cdot / \mathrm{MA}$} &.$/ 1 P$ & V/Er & $r e / 1 \pm \Delta r / P A$ & $r \& / \backslash \pm A V / Y I$ & كنترل & \multirow{4}{*}{ 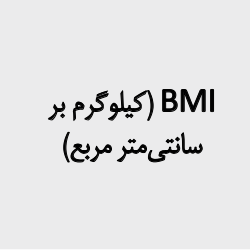 } \\
\hline & & $+1+\infty$ & $r / \pi r$ & $r e / . \pm 1 Y / q \Delta$ & $r \varepsilon / . \pm q r / q$ & تمرين & \\
\hline & &.$M 11$ & $1 / 1$ & $W W / \perp \pm \& \Delta /{ }^{\prime} \Delta$ & $r N \backslash \pm \cdot F / q T$ & مكمل مل & \\
\hline & & $\cdot 1 \cdot r^{*}$ & $P / \Delta$. & $r e / 1 \pm q 1 / R$ & $W / \backslash \pm \Delta V / q)$ & مكمل-تمرين & \\
\hline \multirow{4}{*}{. /Ar } & \multirow{4}{*}{$+/ v 8$} & $\cdot|A|$ &.$- / M$ & $* \pm \lambda r / \cdot \gamma$ & $\cdot / \cdot \pm \lambda r / \cdot V$ & كنترل & \multirow{4}{*}{ WHR (سانتىمتر) WHR } \\
\hline & &.$/ 19$ & $1 / \pi T^{2}$ & $* / * \pm \lambda \mathrm{V} / * \varphi$ & $\cdot / \cdot \pm \lambda Y / \cdot r$ & تمرين & \\
\hline & &.$/ 4$ & $+1+r$ & $* \pm v q \cdot r$ & $\cdot 1 \cdot \pm r q / \cdot r$ & مكمل & \\
\hline & &.$/ M P$ & I/Te & $\cdot 1 \cdot \pm \lambda \mid / \cdot \Delta$ & $.1 . \pm \lambda r / .8$ & مكمل-تمرين & \\
\hline
\end{tabular}


سرمى، ترى كليسريد و HDL به تحقيقات بيشترى نياز است. نتيجليرى

با توجه به نتايج بهدست آمده به نظر مىرسد زنان داراي اضافه

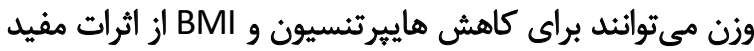

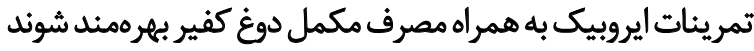

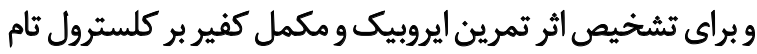

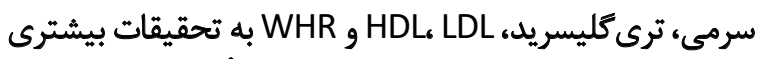

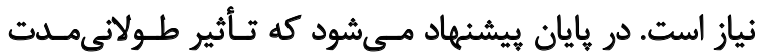

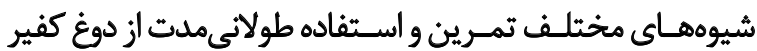

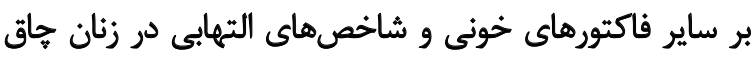

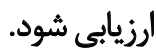

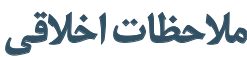

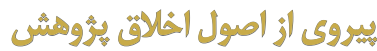

اين مطالعه با كد اخلاق IR.QOM.REC.1398.01 در كميته اخلاق يثروش دانشعاه قم به ثبت رسيده است.

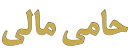

اين مقاله حاصل بخشى از نثايج باياننامه كارشناسى ارشد

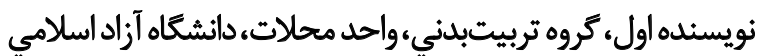

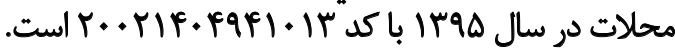

\section{مشار كت نويسندكان}

تمامى نويسندكان معيارهاى استاندارد نويسندكى بر اساس

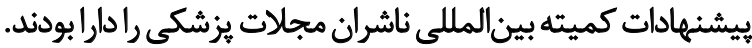

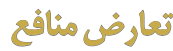

نويسندكان بدينوسيله تصريح مي كنئد كه هيجّمونه تضاد منافعى در خصوص يُروهش حاضر وجود ندارد.

$$
\text { 管 }
$$

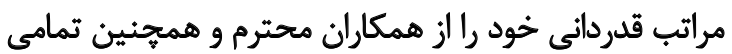

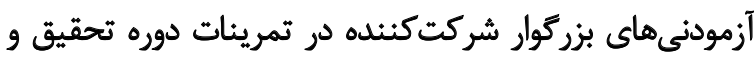

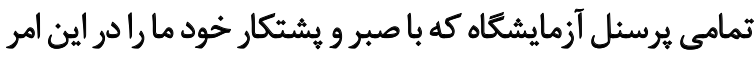

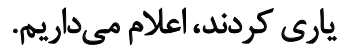

است؛ همجنين نتايج يُروهش حاضر با يافتههاى يُروهشكَرانى

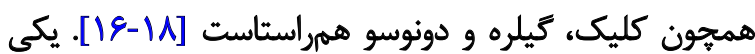

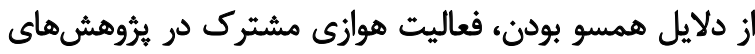

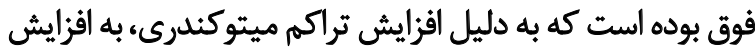

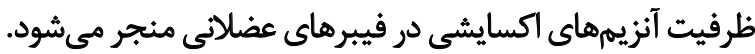

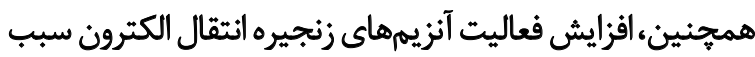

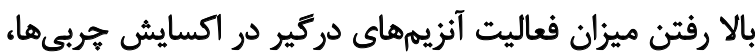

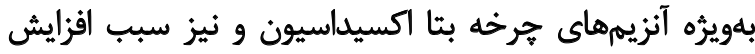

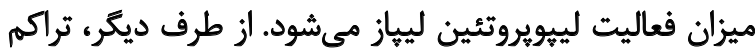

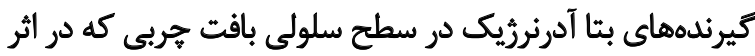

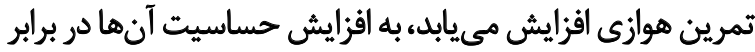

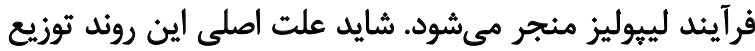

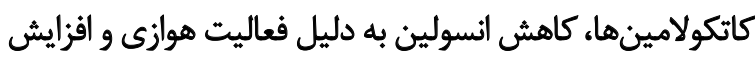

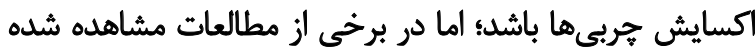

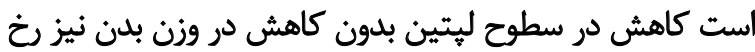

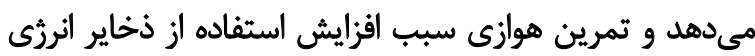

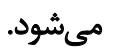

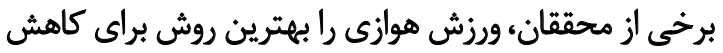

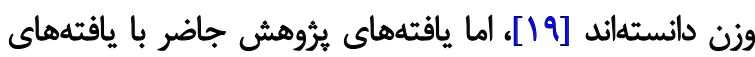

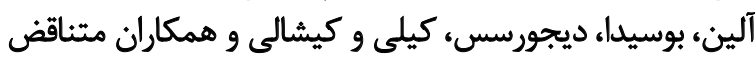

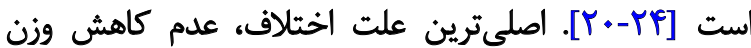

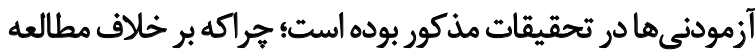

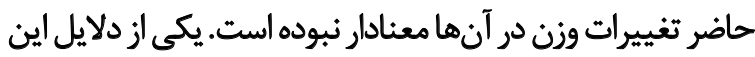

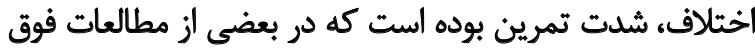

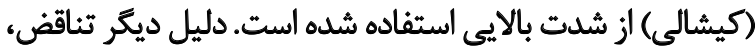

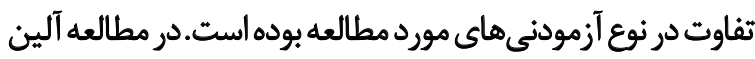

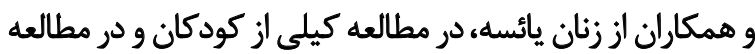

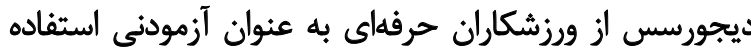

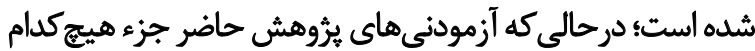

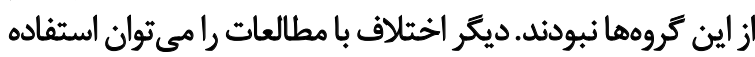

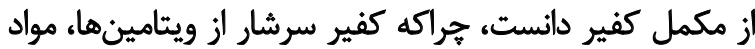

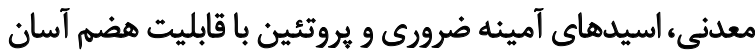

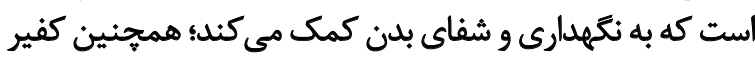

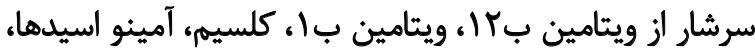

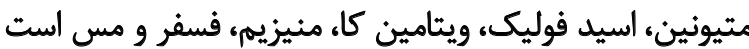

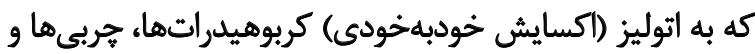
يروتئينها و رشد سلولها و توليد انرثى كمك مى كند.

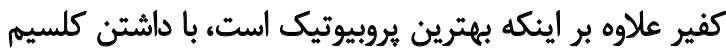

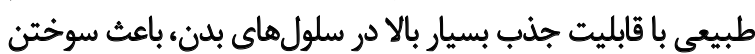

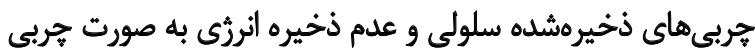

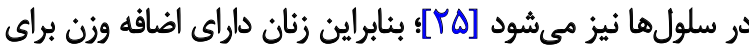

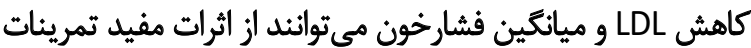

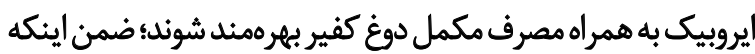
براى تشخيص اثر تمرين ايروبيك و مكمل دوغ كفير بر كلسترول ثنام 


\section{References}

[1] Rains TM, Agarwal S, Maki KC. Antiobesity effects of green tea catechins: A mechanistic review. J Nutr Biochem. 2011; 22(1):1-7. [DOI:10.1016/j. jnutbio.2010.06.006] [PMID]

[2] Dridi S, Taouis M. Adiponectin and energy homeostasis: Consensus and controversy. J Nutr Biochem. 2009; 20(11):831-9. [DOI:10.1016/j.jnutbio.2009.06.003] [PMID]

[3] Kimiagar M, Noori N, Esmaillzadeh A. [The effect of weight loss on waist circumference and hip circumference of overweight and obese women (Persian)]. Iran J Endocrinol Metab. 2005; 7(3):255-61. http://ijem. sbmu.ac.ir/article-1-325-en.html

[4] Salminen S, Bouley C, Boutron MC, Cummings JH, Franck A, Gibson GR, et al. Functional food science and gastrointestinal physiology and function. Br J Nutr. 1998; 80(S1):S147-S71. [DOI:10.1079/BJN19980108] [PMID]

[5] Lopitz-Otsoa F, Rementeria A, Elguezabal N, Garaizar J. [Kefir: A symbiotic yeasts-bacteria community with alleged healthy capabilities (Spanish)]. Rev Iberoam Micol. 2006; 23(2):67-74. [DOI:10.1016/S11301406(06)70016-X

[6] Jürimäe J, Hofmann $P$, Jürimäe $T$, Mäestu J, Purge $P$, Wonisch $M$, et al. Plasma adiponectin response to sculling exercise at individual anaerobic threshold in college level male rowers. Int J Sports Med. 2006; 27(4):272-7. [DOI:10.1055/s-2005-865661] [PMID]

[7] Zoladz JA, Duda K, Konturek SJ, Sliwowski Z, Pawlik T, Majerczak J. Effect of different muscle shortening velocities during prolonged incremental cycling exercise on the plasma growth hormone, insulin, glucose, glucagon, cortisol, leptin and lactate concentrations. J Physiol Pharmacol. 2002; 53(3):409-22. [PMID]

[8] Stucchi AF, Terpstra AHM, Foxall TL, Nicolosi RJ, Smith SC. The effect of exercise on plasma lipids and LDL subclass metabolism in miniature swine. Med Sci Sports Exerc. 1991; 23(5):552-61. [DOI:10.1249/00005768199105000-00007] [PMID]

[9] Khaksari M, Ahmadi M, Najafipour H, Shahrokhi N. Effect of Bunium persicum aqueous extract plus endurance exercise on cardiorespiratory capacity and serum lipid profile. Avicenna J Phytomed. 2014; 4(2):118-26. [PMID] [PMCID]

[10] Seyhaneyildiz Can S, Kutluyer F, Can E, Kayiş Ş, Delihasan Sonay F, Köse Ö, et al. Effect of dietary kefir on the digestive and liver enzymes activities, and glucose level of Coruh trout, Salmo coruhensis (Actinopterygii: Salmoniformes: Salmonidae. Acta Ichthyol Piscat. 2014; 44(2):167-70. [DOI:10.3750/AIP2014.44.2.13]

[11] West NP, Pyne DB, Peake JM, Cripps AW. Probiotics, immunity and exercise: A review. Exerc Immunol Rev. 2009; 15:107-26. [PMID]

[12] Wilt TJ, Shaukat A, Shamliyan T, Taylor BC, MacDonald R, Tacklind J, et al. Number 192: Lactose intolerance and health. Rockville, MD: Agency for Healthcare Research and Quality (AHRQ); 2010. https:// www.ahrq.gov/downloads/pub/evidence/pdf/lactoseint/lactint. pdf?pagewanted=all

[13] Wang H, Wen Y, Du Y, Yan X, Guo H, Rycroft JA, et al. Effects of catechin enriched green tea on body composition. Obesity. 2010; 18(4):773-9. [DOI:10.1038/oby.2009.256] [PMID]

[14] O'Brien KV, Stewart LK, Forney LA, Aryana KJ, Prinyawiwatkul W, Boeneke CA. The effects of postexercise consumption of a kefir beverage on performance and recovery during intensive endurance training. J Dairy Sci. 2015; 98(11):7446-9. [DOI:10.3168/jds.2015-9392] [PMID]
[15] Bahrami K, Shahdadi A. [The effect of aerobic exercise on serum leptin levels and body mass index in obese young girls (Persian)]. Qom Univ Med Sci J. 2015; 9(6):27-36. http://journal. muq.ac.ir/article-1-223-en.html

[16] Nuri Çelik M, Iri R. Effects of competition term trainings on leptin values of female judokas. Afr J Pharm Pharmacol. 2011; 5(6):683-7. [DOI:10.5897/AJPP11.202]

[17] Donoso MA, Muñoz-Calvo MT, Barrios V, Garrido G, Hawkins F, Argente J. Increased circulating adiponectin levels and decreased leptin/ soluble leptin receptor ratio throughout puberty in female ballet dancers: Association with body composition and the delay in puberty. Eur J Endocrinol. 2010; 162(5):905-11. [DOI:10.1530/EJE-09-0874] [PMID]

[18] Rosa G, Cruz L, De Mello DB, De Sa Rego Fortes M, Dantas EHM. Plasma levels of leptin in overweight adults undergoing concurrent training. Int Sport Med J. 2010; 11(3):356-62. https://journals.co.za/content/ismj/11/3/EJC48395

[19] Dodt C, Lönnroth P, Fehm HL, Elam M. The subcutaneous lipolytic response to regional neural stimulation is reduced in obese women. Diabetes. 2000; 49(11):1875-9. [DOI:10.2337/diabetes.49.11.1875] [PMID]

[20] Jarrete AP, Novais IP, Nunes HA, Puga GM, Delbin MA, Zanesco A. Influence of aerobic exercise training on cardiovascular and endocrine-inflammatory biomarkers in hypertensive postmenopausal women. J Clin Transl Endocrinol. 2014; 1(3):108-14. [DOI:10.1016/j.jcte.2014.07.004] [PMID] [PMCID]

[21] Bouassida A, Chamari K, Zaouali M, Feki Y, Zbidi A, Tabka Z. Review on leptin and adiponectin responses and adaptations to acute and chronic exercise. Br J Sports Med. 2010; 44(9):620-30. [DOI:10.1136/ bjsm.2008.046151] [PMID]

[22] Desgorces FD, Chennaoui M, Gomez-Merino D, Drogou C Guezennec CY. Leptin response to acute prolonged exercise after training in rowers. Eur J Appl Physiol. 2004; 91(5-6):677-81. [DOI:10.1007/s00421-003-1030-0] [PMID]

[23] Kelly AS, Steinberger J, Olson TP, Dengel DR. In the absence of weight loss, exercise training does not improve adipokines or oxidative stress in overweight children. Metabolism. 2007; 56(7):1005-9. [DOI:10.1016/j. metabol.2007.03.009] [PMID]

[24] Kishali NF. Serum leptin level in healthy sedentary young men af ter a short-term exercise. Afr J Pharm Pharmacol. 2011; 5(4):522-6. [DOI:10.5897/AJPP11.096]

[25] Ninane V, Berben G, Romnee JM, Oger R. Variability of the microbia abundance of kefir grain starter cultivated in partially controlled conditions. Biotechnol Agron Soc Environ. 2005; 9(3):191-4. https://popups. uliege.be/1780-4507/index.php?id=1463 